\title{
Inhibition of Dll4/Notch1 pathway promotes angiogenesis of Masquelet's induced membrane in rats
}

\author{
Qian Tang ${ }^{1}$, Haimin Jin ${ }^{1}$, Minji Tong ${ }^{1}$, Gang Zheng ${ }^{1}$, Zhongjie Xie', Shangkun Tang ${ }^{1,3}$, Jialei Jin ${ }^{1,3}$, Ping Shang ${ }^{2}$, \\ Huazi Xu', Liyan Shen', Yu Zhang ${ }^{1}$ and Haixiao Liu (iD ${ }^{1}$
}

\begin{abstract}
The Masquelet's induced membrane technique for repairing bone defects has been demonstrated to be a promising treatment strategy. Previous studies have shown that the vessel density of induced membrane is decreased in the late stage of membrane formation, which consequently disrupts the bone healing process. However, relatively little is known about certain mechanisms of vessel degeneration in the induced membrane tissue and whether promotion of angiogenesis in induced membranes can improve bone regeneration. Here, we showed that the Delta-like ligand 4/ Notch homolog 1 (DII4/Notch1) pathway was relatively activated in the late stage of induced membrane, especially at the subcutaneous site. Then, DAPT, a classical $\gamma$-secretase inhibitor, was applied to specifically inhibit Notch1 activation, followed by up-regulation of vascular endothelial growth factor receptor 2 (VEGFR2) and CD31 expression. DAPTmodified induced membranes were further confirmed to contribute to bone regeneration after autogenous bone grafting. Finally, in vitro experiments revealed that knocking down Notch1 contributed to the functional improvement of endothelial progenitor cells (EPCS) and that DAPT-treated induced membrane tissue was more favorable for angiogenesis of EPCs compared with the vehicle group. In conclusion, the present findings demonstrate that DII4/ Notch1 signaling is negatively associated with the vessel density of induced membrane. Pharmacological inhibition of Notch1 attenuated the vessel degeneration of induced membrane both in vitro and in vivo, which consequently improved bone formation at the bone defect site and graft resorption at the subcutaneous site.
\end{abstract}

\section{Introduction}

The reconstruction of large-size bone defects caused by major trauma, surgical excision of tumors and debridement after posttraumatic septic non-unions or osteitis, is still a challenging problem in orthopedic surgery ${ }^{1-4}$. The

Correspondence: Liyan Shen (uynixmm@163.com) or Yu Zhang (drzhangyu@hotmail.com) or Haixiao Liu (spineliu@163.com)

'Department of Orthopaedic Surgery, The Second Affiliated Hospital and Yuying Children's Hospital of Wenzhou Medical University, 109, Xueyuanxi Road, 325027 Wenzhou, China

2Department of Rehabilitation, The Second Affiliated Hospital and Yuying Children's Hospital of Wenzhou Medical University, 109, Xueyuanxi Road, 325027 Wenzhou, China

${ }^{3}$ Department of Clinical Medicine, Second Clinical Medical College, Wenzhou Medical University, 109, Xueyuanxi Road, 325027 Wenzhou, China

These authors contributed equally: Qian Tang, Haiming Jin. vascularized fibular graft $^{5}$, artificial bone substitutes graft $^{6}$, and the Ilizarov bone transfer ${ }^{7}$ are the commonly used techniques for this issue. However, the induced membrane technique described by Masquelet et al. has emerged as an alternative strategy for the treatment of serious bone defect ${ }^{8,9}$. This technique consists of two stages of surgery: first, a polymethylmethacrylate (PMMA) cement spacer is inserted into the bony defect to create an induced membrane with bone healing properties. Second, after 6-8 weeks of implantation, the spacers were removed followed by autologous bone filling 8 . By using this technique, the defect in tibia, femur, humerus, hand, wrist, ulna, and mandibular are all reported to achieve satisfactory bone healing ${ }^{10-14}$. 
Most previous researches of Masquelet's technique focused on the combined application of this induced membrane with diverse graft substitutes or growth factors ${ }^{15-17}$, but few studies target the improvement of membrane itself. Actually, the Masquelet's induced membrane is composed of fibrous inner layer (closest to the PMMA cement) and an outermost vascularized layer (furthest from the cement) ${ }^{18}$. This vascularized membrane not only inhibit soft tissue invasion to protect grafts from resorption, but also support bone formation via its blood supply. This function is similar to vascularized periosteum acts in bone defect repair or fracture healing. The membrane could promote corticalization of the cancellous bone even in bad vascularized bed-like irradiated tissue or in an infrequent bone disease like congenital pseudarthrosis ${ }^{11,18-20}$. However, our previous study performed in rabbit as well as the work of Henrich et al. and Aho et al. revealed that the vessel density of membrane is decreased after 6 weeks of membrane formation and the membranes in subcutaneous showed less microvessels than those in bone defect ${ }^{11,21,22}$. What's more, due to the surrounded vascular axes, the membrane formed in femoral defect and humeral defect was less vascularized and in lower quality than which in tibia defects. It would affect the successful rate of secondary operation? 9 Thus, it is favorable to clinic application by promoting angiogenesis during the induced membrane formation.

It has become clear that Dll4/Notch1 signaling pathway exerts a regulatory role in the proliferation and differentiation of endothelial cells ${ }^{23,24}$. In postnatal tissues, Notch signaling favored the endothelium in quiescent state by inhibiting endothelial cell proliferation and cell-cell contact ${ }^{25}$. Besides, it also suppresses endothelial tip cell formation and vessel branching ${ }^{26}$. There are four Notch receptors (Notch 1, 2, 3, and 4) and five Notch ligands (Delta-like1, 3, 4 (Dll1,3,4), Jagged 1, 2) found in mammals ${ }^{27}$. Among these molecules, the Dll4 and Notch 1 are detected predominantly in the endothelium and Dll4 is particularly found only in the endothelium ${ }^{25,28,29}$. It was reported that inhibition of Notch1 signaling by blockade of a Notch signaling enzyme complex (gamma secretase) increased the number of endothelial cells participating in sprouting and consequently increased the vessel density $^{30-32}$. Inhibition of Dll4/Notch1 signaling was shown to improve the flap survival, would healing and attenuated cerebral ischemia via the promotion on neovascularization and angiogenesis ${ }^{33-35}$. In present study, we first investigate the role that Dll4/Notch1 pathway played in Masquelet's induced membrane in both subcutaneous and femur defects. Furthermore, we examined whether inhibition of Dll4/Notch1 could promote the angiogenesis in this membrane and whether it could consequently improve the bone formation. Finally, we also determined whether this modified membrane promote the angiogenesis in endothelial progenitor cells in vitro.

\section{Materials and methods \\ Reagents and antibodies}

Gamma secretase inhibitor IX (also named DAPT (N[N-(3,5-Difluorophenacetyl-L-alanyl)]-S-phenylglycine tButyl Ester) and dimethylsulfoxide (DMSO) were purchased from Sigma-Aldrich (St Louis, MO, USA). The primary antibody against Hes1, CD3, PCNA, SDF-1, and $\beta$-actin were acquired from Abcam (Cambridge, UK), Dll4 and ICAM-1 antibodies was obtained from Novus (Littleton, CO, USA), goat anti-rabbit, and anti-mouse IgGHRP were from Bioworld (OH, USA) and antibodies against Notch1 and VEGFR2 were purchased from Cell Signaling Technology (Danvers, MA, USA); Alexa Fluor ${ }^{\circledR} 488$ labeled and Alexa Fluor ${ }^{\circledR} 594$ labeled Goat Anti-Rabbit IgG $(\mathrm{H}+\mathrm{L})$ second antibody was purchased from Jackson ImmunoResearch (West Grove, PA, USA). The 4',6-diamidino-2-phenylindole (DAPI) was obtained from Beyotime (Shanghai, China). The cell culture reagents were purchased from Gibco (Grand Island, NY, USA).

\section{Animal model}

Ninety male Sprague-Dawley rats with a mean weight of $350 \mathrm{~g}$ were purchased from Animal Center of Chinese Academy of Sciences Shanghai, China. The protocol for animal care and use was according to the Guide for the Care and Use of Laboratory Animals of the National Institutes of Health and was approved by the Animal Care and Use Committee of Wenzhou Medical University (ethic code: wydw2014-0129). Rat critical-sized femur segmental defects were created in the right femur by using a model according to Henrich et al. ${ }^{18}$ After anesthetized with intraperitoneally injection of $2 \%(\mathrm{w} / \mathrm{v})$ pentobarbital $(40 \mathrm{mg} / \mathrm{kg})$, the rats were placed in the left lateral prone position, and a $40 \mathrm{~mm}$ longitudinal incision was made over the lateral aspect of the right thigh. Then, the biceps femoris and vastus lateralis muscles were separated to expose the lateral aspect of the femoral bone. A six-hole, $1.0 \mathrm{~mm}$ titanium mini-plate (F215002.T01, Fengyi, Tianjin, China) was applied to the lateral aspect of the femur shaft and secured in place with four $1.5 \mathrm{~mm}$ cortical screws. A critical-sized defect (CSD) measuring $10 \mathrm{~mm}$ was induced via using a reciprocating saw followed by filling with a $10 \mathrm{~mm}$ PMMA cement cylinder, which molded ex vivo. The wound was irrigated with sterile saline, the muscles as well as the fascia were carefully reapproximated with 4-0 Vicryl sutures and the skin was closed with 3-0 silk suture. Meanwhile, the PMMA spacers were also inserted into subcutaneous in the left leg to create a comparable subcutaneous model. The rats were divided into two groups after surgery: DAPT 
Table 1 Primer sequences used in qRT-PCR

\begin{tabular}{|c|c|c|}
\hline Gene & Forward primer & Reverse primer \\
\hline DLL1 & 5'- CACGAGAAAACCAGAAAGC-3' & 5'- ATGCCCGGAAAGTCTATGTG-3' \\
\hline DLL3 & 5'- ACTGGATGCCTIITACCTGG -3' & 5'-ACATCGAAGCCCGTAGAATC-3' \\
\hline DLL4 & 5'- CTITACCGGCTCTAACTGTGAG-3' & 5'-GGCTTGGACCTCTGTTCAG-3' \\
\hline Jagged1 & 5'- GGTGGACAGCTCTGTGACAA-3' & 5'-CAGCCTGGAGAACACTCACA-3' \\
\hline Jagged2 & 5'-ACGAGGAGGATGAAGAGCTGA-3' & 5'-GGGGTCTTTGGTGAACTTGTG-3' \\
\hline Notch1 & 5'- TGAGACTGCCAAAGTGTTGC-3' & 5'- GTGGGAGACAGAGTGGGTGT-3' \\
\hline Notch2 & 5'-CCTGAACGGGCAGTACATTT-3' & 5'-GCGTAGCCCTTCAGACACTC-3' \\
\hline Notch3 & 5'-ATACACTGGCCCCTTCTGTG-3' & 5'-GTCGAGGCAAGAACAGGAAA-3' \\
\hline Notch4 & 5'-CTCTGTCCGCCTTCTTTCTG-3' & 5'-CAAGCACACACCTCCGTTG-3' \\
\hline$\beta$-actin & 5'- AGCCATGTACGTAGCCATCC-3' & 5'- GCTGTGGTGGTGAAGCTGTA-3' \\
\hline
\end{tabular}

administration group (50ng/kg, iv, dissolved in $1 \%$ DMSO in saline) and vehicle group (1\% DMSO in saline). Thirty rats that received different treatments were killed at 6 week after surgery for in vitro study, another thirty rats were killed at 6 week to examine the characteristics of the induced membrane in different administration. And, the rest thirty rats were received the secondary operation to evaluate the osteogenic ability at 6 week after surgery. In brief, after carefully removing the PMMA spacers, autologous corticocancellous bone graft collected from two caudal vertebrae, which obtained by amputation of the distal third of the tail, were used to fill the cavity. Then, the membrane, muscle, and skin were closed by 4-0 Vicryl sutures or 3-0 silk sutures successively.

\section{In vivo study \\ Histology}

Induced membrane samples collected at 6 week after first-stage surgery and the bone site samples were collected at 3 month after secondary operation. Samples containing bone tissue were additionally demineralized in a 10\% EDTA solution. All samples were fixed in $4 \%(\mathrm{v} / \mathrm{v})$ neutral paraformaldehyde for $24 \mathrm{~h}$ after collection. Following dehydrated and embedded in paraffin, the tissues were cut into $5-\mu \mathrm{m}$ sagittal sections. Then, the slides were stained with hematoxylin and eosin and bone tissue slides were additionally stained with Masson's staining. The figures were examined and captured by another group of experienced histology researchers in a blinded manner using a microscope (Olympus, Japan). There were 15 specimens in each group and five randomly selected fields in each slide were used for quantitative analysis by using Image-Pro Plus software, version 6.0 (Media Cybernetics, Rockville, MD, USA).

\section{Real-time polymerase chain reaction}

Total RNA $(0.5 \mu \mathrm{g})$ was extracted from the different membrane tissue using TRIzol reagent (Invitrogen) according to the manufacturer's protocol. First-strand cDNA was synthesized by a reverse transcriptase kit (MBI Fermantas, Germany) according to the manufacturer's instructions. Real-time amplification, using SYBR Green Supermix (QPK-212, Tokyo, Japan) and CFX96 RealTime PCR System (Bio-Rad Laboratories, CA, USA). The qPCR conditions were as follows: $10 \mathrm{~min} 95^{\circ} \mathrm{C}$, followed by 40 cycles of $15 \mathrm{~s} 95^{\circ} \mathrm{C}$ and $1 \mathrm{~min} 60^{\circ} \mathrm{C}$. $\beta$-actin was used as an internal control. The cycle threshold $(\mathrm{Ct})$ values were collected and normalized to the level of GAPDH. The level of relative mRNA of each target gene was calculated by using the $2^{-\Delta \Delta \mathrm{Ct}}$ method. The primers of Dll1, Dll3, Dll4, Jagged1, Jagged2, Notch1, Notch2, Notch3, and Notch4 were designed with the aid of NCBI Primer-Blast Tool (https://www.ncbi.nlm.nih.gov/tools/primer-blast/), which were listed in Table 1.

\section{X-ray imaging method}

Thirty rats after 3 month of secondary operation within or without DAPT treatment before were given the X-ray examination. X-ray imaging was performed on all rats to evaluate the bone growth, resorptions, and bridging callus formation between fracture end and grafts by using a digital X-ray machine (Kubtec Model XPERT.8; KUB Technologies Inc.). Proper images were obtained in the following settings: $50 \mathrm{kV}$ and $160 \mu \mathrm{A}$.

\section{Protein extraction from induced membranes}

The induced membranes collected from subcutaneous site and bone defect site were rapidly stored at $-80^{\circ} \mathrm{C}$ for western blotting. Briefly, frozen animal membrane tissues homogenized in ice-cold RIPA lysis buffer (containing 50 $\mathrm{mM}$ Tris- $\mathrm{HCl} \mathrm{pH}$ 8.0, $150 \mathrm{mM} \mathrm{NaCl}, 1 \% \mathrm{NP}-40,0.5 \%$ 
deoxycholate, $0.1 \%$ SDS, $10 \mathrm{mM} \mathrm{Na}_{2} \mathrm{P}_{2} \mathrm{O}_{7}, 10 \mathrm{mM} \mathrm{NaF}, 1$ $\mathrm{mg} / \mathrm{ml}$ aprotinin, $10 \mathrm{mg} / \mathrm{ml}$ leupeptin, $1 \mathrm{mM}$ sodium vanadate) and $1 \mathrm{mM}$ PMSF (phenylmethanesulfonyl fluoride). Tissue homogenates were incubated for $15 \mathrm{~min}$ at $4{ }^{\circ} \mathrm{C}$ and centrifuged at $12,000 \mathrm{rpm}$, for $15 \mathrm{~min}$ at $4{ }^{\circ} \mathrm{C}$. The supernatant containing the soluble proteins was recovered. Total proteins in tissue lysates were quantified using the BCA Protein Assay Kit (Beyotime Biotechnology, Shanghai, China) according to the manufacturer's protocol. The quantified protein solutions are used for western blot analysis and in vitro experiments.

\section{Western blotting}

The equivalent of $70 \mu \mathrm{g}$ of total protein was loaded onto SDS-PAGE and transferred to PVDF membrane (BioRad). The membrane was blocked with 5\% non-fat milk in TBS with $0.1 \%$ Tween 20 for $90 \mathrm{~min}$ and then incubated overnight at $4{ }^{\circ} \mathrm{C}$ with the primary antibody against Dll4 (1:250), Notch1 (1:1000), Hes1 (1:1000), VEGFR2 (1:1000), CD31 (1:500), and $\beta$-actin (1:1000) according to the manufacturer's recommendations. Then, the membranes were washed with TBST for 5 min three times, and primary antibodies were detected with horseradish peroxidase-conjugated secondary antibodies. The bands were visualized and quantitated using the Image Lab 3.0 software (Bio-Rad).

\section{Immunohistochemical examination}

The sections embedded in paraffin were deparaffinized and rehydrated and then the endogenous peroxidase activity was need to be blocked by $3 \%$ hydrogen peroxide. After that, the sections were incubated with $0.4 \%$ pepsin (Sangon Biotech, Shanghai, China) in $5 \mathrm{mM} \mathrm{HCl}$ at $37^{\circ} \mathrm{C}$ for $20 \mathrm{~min}$ for antigen retrieval and nonspecific-binding sites were blocked by $10 \%$ bovine serum albumin for 30 min at room temperature. The sections were then incubated with the primary antibody (anti-Notch1, 1:400, antiCD31, 1:100) overnight at $4{ }^{\circ} \mathrm{C}$. Finally, the sections were incubated with an appropriate HRP-conjugated secondary antibody (Santa Cruz Biotechnology, Dallas, TX, USA). Images were saved using Image-Pro Plus software, version 6.0 (Media Cybernetics, Rockville, MD, USA), and the integral absorbance values were used as indicators of Notch1 expression levels. Meanwhile, the numbers of CD31-positive blood vessels per unit area $\left(\mathrm{mm}^{2}\right)$ were calculated. At least five random fields of three random sections from each tissue sample were used to quantify the positive cells.

\section{In vitro experiment \\ Primary endothelial progenitor cells (EPCS) isolation, cultiva- tion, and determination}

EPCs were isolated from the bone marrow of rats. Briefly, mononuclear cells were isolated from the tibia and femur of male SD rats (4 weeks of age) via using Percoll density gradient centrifugation. Then, the cells were cultured on human fibronectin-coated flask in endothelial cell growth media (EGM-2) supplemented with EGMTM2 MV SingleQuotsTM (Lonza, Basel, Switzerland), rat recombinant vascular epidermal growth factor $(20 \mathrm{ng} / \mathrm{ml})$, insulin-like growth factor $(4 \mathrm{ng} / \mathrm{ml})$, fibroblast growth factor $(4 \mathrm{ng} / \mathrm{ml})$, and $5 \%$ fetal bovine serum. After culturing in a $5 \% \mathrm{CO}_{2}$ incubator for 3 days, non-adherent cells were removed by washing with phosphate-buffered saline (PBS). The resident cells were incubated in fresh medium for a further 3 days. When up to $80-90 \%$ confluency, the cells were harvested by using $0.25 \%$ TrypsinEDTA (Gibco, Invitrogen). Then, cells were replanted into $10 \mathrm{~cm}$ culture plates at the appropriate density and the complete medium was changed every 3 days. The third passage EPCs were then used for the following experiments.

The phenotype of EPCs was first determined by fluorescence-activated cell sorter (FACS) analysis to assess the expression of endothelial markers in the EPCs population. Briefly, cells were detached using trypsin-EDTA and gentle scraping. Then, cells incubated with saturating concentrations of the following monoclonal antibodies: fluorescein-labeled (FITC) CD31, VEGFR2, vascular endothelial (VE)-cadherin, endothelial nitric oxide synthase (eNOS), and the monocytic marker $\mathrm{CD} 45$ as a control, for $30 \mathrm{~min}$ at $4{ }^{\circ} \mathrm{C}$ in the dark. Using a FACSCalibur flow cytometer (Becton-Dickinson, San Jose, CA, USA), quantitative analysis was performed to measure $1 \times 10^{5}$ cells/sample. EPCs were defined as those cells that were positive for CD31, VEGFR2, VE-cadherin, and Enos with low cytoplasmatic granularity. What is more, the phenotype also determined according to both Dil-acLDL (Biomedical Technologies, Stoughton, MA, USA) uptake and FITC-labeled UEA-1 lectin (Vector Lab, Burlingame, CA, USA) binding. Cells were first incubated with Dil-acLDL at $37^{\circ} \mathrm{C}$ for $4 \mathrm{~h}$ and then fixed with $1 \%$ paraformaldehyde for $10 \mathrm{~min}$. After washing twice, the cells were incubated with FITC-conjugated UEA-1 $(10 \mu \mathrm{g} /$ $\mathrm{ml}$ ) for $1 \mathrm{~h}$. After staining, cells were observed by fluorescence microscopy. In addition, the immunofluorescence staining of CD31 and VEGFR2 were also performed for phenotype determination. The samples were rinsed three times in PBS before fixation using 4\% paraformaldehyde and followed by permeation using the $0.1 \%$ Triton X-100 diluted in PBS for $15 \mathrm{~min}$. Then, the cells were blocked with $5 \%$ bovine serum albumin for $1 \mathrm{~h}$ at $37^{\circ} \mathrm{C}$, rinsed with PBS and incubated with primary antibodies, which diluted in PBS: CD31 (1:200) and VEGFR2 (1:100) in a humid chamber overnight at $4{ }^{\circ} \mathrm{C}$. On the next day, the glass plates were washed and incubated with Alexa Fluor $^{\circledR} 488$ labeled or Alexa Fluor ${ }^{\circledR} 594$ conjugated second antibodies (1:400) for $1 \mathrm{~h}$ at room temperature and 
labeled with DAPI for $5 \mathrm{~min}$. Finally, the image of each slides was chosen randomly for observation with a fluorescence microscope.

\section{Cell administration}

EPCs were seeded at a density of $5 \times 10^{4}$ cells $/ \mathrm{cm}^{2}$ and were cultured in completed medium plus $100 \mu \mathrm{g}$ of protein extract from induced membranes obtained from different groups at 6 week after surgery. After $24 \mathrm{~h}$ adminstration, the cells were collected and used for western blot analysis and capillary-like tube formation assay.

\section{siRNA transfection}

siRNA for rat Notch1 gene was designed and synthesized (RiboBio, Guangzhou, China) with the following sequence: 5'-AAGTGGGACCTGCCTGAATGG-3'. EPCs were seeded in a six-well plate and cultured for $24 \mathrm{~h}$ to $60-70 \%$ confluency. The cells were transfected with 50 nM negative control or siRNA duplexes using Lipofectamine 2000 siRNA transfection reagent (Thermo Fisher, UT, USA).

\section{Western blotting}

The total protein extracted from EPCs was isolated using RIPA lysis buffer with $1 \mathrm{mM}$ PMSF (phenylmethanesulfonyl fluoride) and on the ice for $10 \mathrm{~min}$ followed by $15 \mathrm{~min}$ centrifugation at $12,000 \mathrm{rpm}$ and $4{ }^{\circ} \mathrm{C}$, and then protein concentration was measured using the $\mathrm{BCA}$ protein assay kit (Beyotime). Aliquot of $40 \mathrm{ng}$ of protein was separated by sodium dodecylsulfate-polyacrylamide gel electrophoresis (SDS-PAGE) and transferred to a PVDF membrane (Bio-Rad, USA). After blocking with 5\% non-fat milk for $2 \mathrm{~h}$, the membranes were incubated with the primary antibody against PCNA (1:5000), ICAM-1 (1:500), SDF-1 (1:1000), and $\beta$-actin (1:5000) overnight at $4{ }^{\circ} \mathrm{C}$, and followed by subsequently incubation with respective secondary antibodies for $2 \mathrm{~h}$ at room temperature. After three times washing with TBST, the bands were visualized and quantitated using the Image Lab 3.0 software (Bio-Rad).

\section{Cell migration assay}

The migratory ability of EPCs was assessed using a modified Boyden chamber assay (Transwell; Coster, Cambridge, MA, USA) ${ }^{31}$. First, the third passage of EPCs was placed in the upper chambers of 24-well transwell plates with polycarbonate membrane that contained serum-free endothelial growth medium. The $100 \mu \mathrm{g}$ of protein extract from induced membrane of different treatment groups were added to the $250 \mu \mathrm{l}$ medium in the lower chambers. After co-incubation for $24 \mathrm{~h}$, the membranes of upper chamber were washed with PBS and fixed with $4 \%$ paraformaldehyde. The upper surface of the membrane was carefully wiped with a cotton ball. The membrane was then stained with crystal violet solution. Migration of EPCs was observed by microscopy (magnification of $\times 100$ ). Five random fields of view were used for counting the number of migrated cells

\section{Capillary-like tube formation assay}

The in vitro capillary-like tube formation assay was conducted using ECMatrix ${ }^{\mathrm{TM}}$ (BD Biosciences, German) according to the manufacturer's instructions. Afer thawing on $4{ }^{\circ} \mathrm{C}$ overnight, the ECMatrix ${ }^{\mathrm{Tm}}$ solution was mixed with $10 \times$ ECMatrix $^{\text {ma }}$ diluents and placed in a 96-well tissue culture plate $(50 \mu \mathrm{l} /$ well $)$ at $37^{\circ} \mathrm{C}$ for $1 \mathrm{~h}$ to allow the matrix solution to be solid. The EPCs after $24 \mathrm{~h}$ administration were transferred to be cultured on ECMatrix $^{\mathrm{TM}}$ covered 96-well plate at the density of $1.2 \times$ $10^{4}$ cells $/ \mathrm{ml}$. Tube formation of each group was photographed from five random microscopic fields using an inverted light microscope (Leica, German) and the average numbers of tubes per $\times 40$ magnified field were determined.

\section{Cell invasion assay}

The invasion assay was done by using ECMatrix ${ }^{\mathrm{TM}}$ and Transwell chamber. First, the filter inner of Transwell was coated by ECMatrix ${ }^{\mathrm{TM}}$. Then, EPCs were cultured in the upper chambers and the protein from induced membrane of different treatment groups were added to the $250 \mu \mathrm{l}$ medium in the lower chambers. After co-incubation for $24 \mathrm{~h}$, the membranes of upper chamber were washed with PBS and fixed with $4 \%$ paraformaldehyde. The upper surface of the membrane was carefully wiped with a cotton ball. The membrane was then stained with DAPI solution. Invasive EPCs were observed by microscopy (magnification of $\times 100$ ). Five random fields of view were used for counting the number of invasive cells.

\section{Statistical analysis}

The experiments were at least performed five times. The results were presented as mean \pm SD. Statistical analyses were performed using SPSS statistical software program 20.0. Data were analyzed by one-way analysis of variance (ANOVA) followed by the Tukey's test for comparison between control and treatment groups. $P$ values less than 0.05 were considered significant.

\section{Results}

Animal model and characteristics of induced membranes from subcutaneous site and femoral defect site

Figure 1 illustrated the procedure of the establishment of Masquelet's induced membrane in both subcutaneous site (Fig. 1a-c) and femoral defect site (Fig. 1d-f). The $\mathrm{H} \& \mathrm{E}$ staining results of membrane tissue collected at 2, 4, and 6 weeks after surgery showed similar morphology 


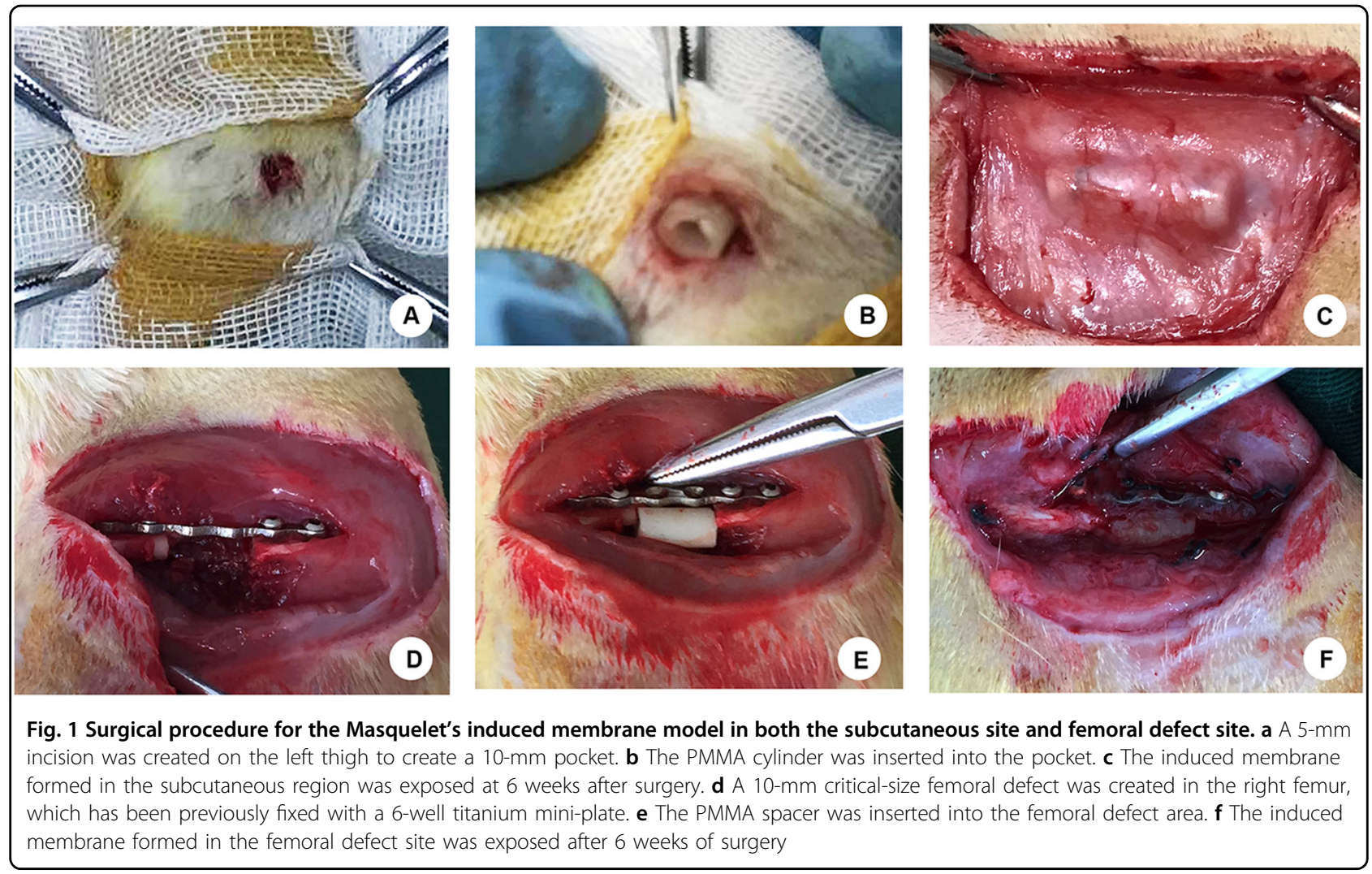

between the membranes induced around femoral defect and induced ectopically in a subcutaneous pocket (Fig. 2a). The cells of inner layer which close to PMMA spacer were in relative regular shape and connect compactly with each other. Whereas, the cells of outer layer were in diverse kinds, loose connective and along with microvessels ingrowth. According to the histomorphometric results, the thickness of induced membranes was increased gradually from 2 to 6 weeks (Fig. 2b, subcutaneous site, 2 weeks: $24.20 \pm 6.93$, 4 weeks: $42.84 \pm$ 10.276 weeks: $85.83 \pm 15.82$; femoral defect site, 2 weeks: $116.76 \pm 9.89,4$ weeks: $140.72 \pm 17.73$, 6 weeks: $164.83 \pm$ 20.48). In contrast, there is a decreasing trend of mean vessel density in both two groups (Fig. 2c, subcutaneous site, 2 weeks: $16.25 \pm 3.28$, 4 weeks: $12.37 \pm 2.286$ weeks: $5.27 \pm 2.24$; femoral defect site, 2 weeks: $46.30 \pm 10.86$, 4 weeks: $34.72 \pm 7.82$, 6 weeks: $24.83 \pm 6.93)$. Meanwhile, the inner layer was significantly thicker and the outer layer possessed more micro-vessels in femoral defect area than those in subcutaneous area at each time point, respectively (all $p<0.01$ ).

\section{Dll4/Notch1 pathway in the induced membrane of subcutaneous site and bone defect site}

To investigate the potential role of Notch signaling in vessel degeneration during induced membrane formation, we analyzed the expression levels of nine relative ligands and receptors involved in Notch signaling by qRT-PCR. The results showed that Dll4 and Notch1 were most significantly upregulated during membrane formation and both of them expressed lower in membrane from femoral defect site than it in subcutaneous (Fig. 3a). Then, as is shown in Fig. 3b, c, the femoral induced membrane showed lower protein expression of Dll4, Notch intracellular domain (NICD) of Notch1 and Hairy and enhancer of split-1 (Hes1) but higher expression level of vascular endothelial growth factor receptor 2 (VEGFR2) and CD31(vessel marker) than membrane formed in subcutaneous site at 6 weeks. Besides, similar result was revealed by immumohistochemical staining of Notch1. The Notch1-positive area was significantly lower in both inner layer and outer layer of membranes in bone defect area than those in subcutaneous area (Fig. 3d). In addition, the quantitative analysis of integral absorbance of Notch1 protein was consistent with macrographic results (Fig. 3e).

\section{Inhibition of Dll4/Notch1 pathway promotes angiogenesis in induced membranes of subcutaneous site and bone defect site}

To investigate whether inhibition of Notch1 could promote the angiogenesis of induced membrane, we treated the rats after surgery by DAPT during the 6 weeks of membrane formation, which is a classical 


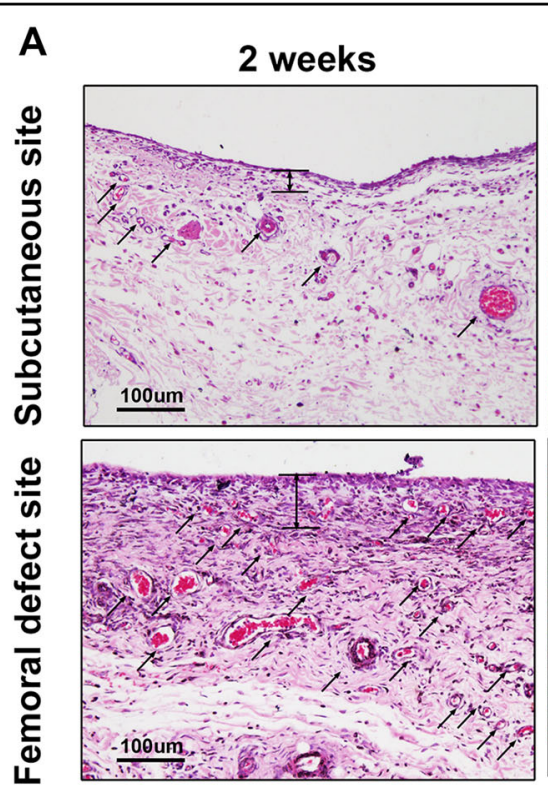

B

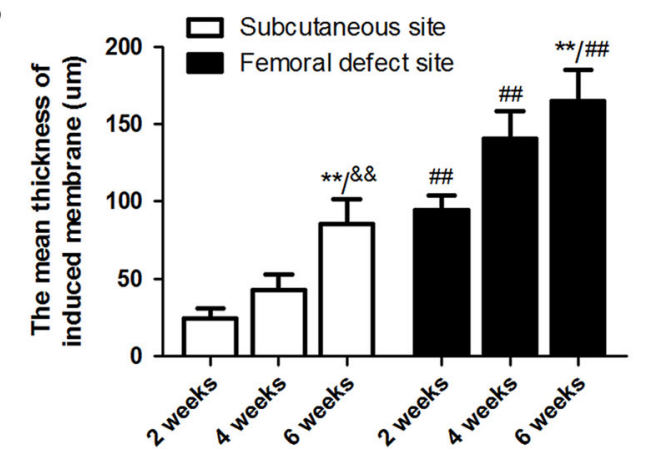

4 weeks

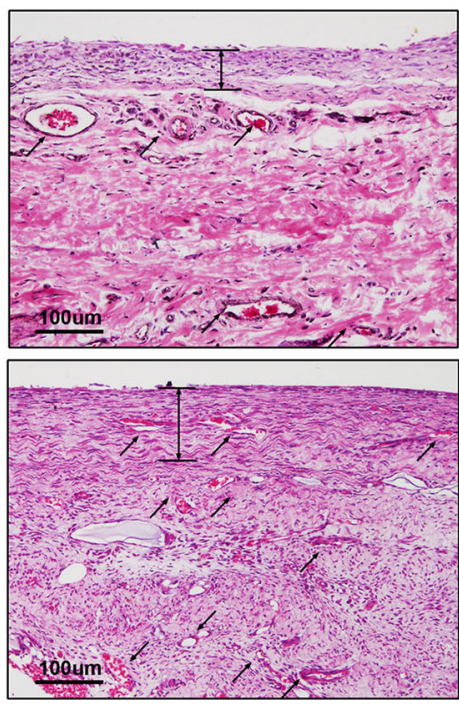

6 weeks
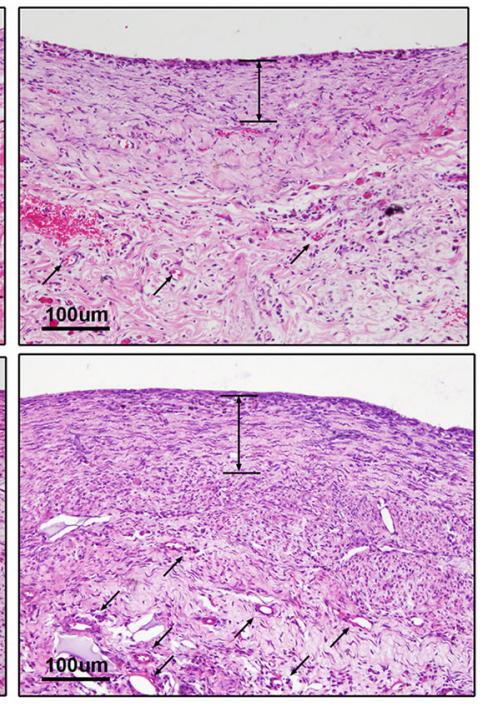

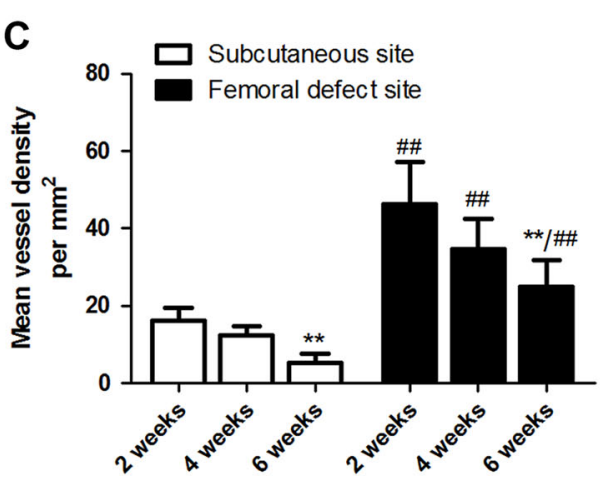

Fig. 2 Characteristics of the induced membrane formed in the subcutaneous site and femoral defect site. a H\&E results of the induced membrane formed in the subcutaneous site and femoral site at 2, 4, and 6 weeks (scale bar: $100 \mu$ m, black arrows indicate microvessels). $\mathbf{b}$ Quantitative results for membrane thickness. c Quantitative results of vessel density in induced membranes. The data in the figures represent averages \pm S.D. Significant differences between different groups are indicated as ** $P<0.01$, compared with 2-week induced membrane in the subcutaneous site and femoral defect site, respectively; ${ }^{\&} P<0.01$, compared with 4 -week induced membrane in the subcutaneous site; ${ }^{\# \# ~} P<0.01$ compared with the same time point for induced membrane in the subcutaneous site; for each group, $n=5$

$\gamma$-secretase inhibitor targeting Notch1(NICD) expression selectively. According to the Fig. 4a, b, although the Dll4 expression level was not altered after DAPT administration in both femoral and subcutaneous induced membrane, the expression level of Notch1(NICD) and Hes1 were significantly inhibited by DAPT, which consequently upregulated the VEGFR2 and CD31 expression. What is more, immumohistochemical staining results of Notch1 also revealed an inhibitory effect of DAPT (Fig. 4c, d) and the number of CD31-positive vessels were determined to be formed more in DAPT treatment groups compared with vehicle group in both two induced environments (Fig. 5a, b. Subcutaneous site: $8.33 \pm 4.04$ (vehicle) vs. $25.06 \pm 3.46$ (DAPT), $p<0.01$; femoral defect site: $28.67 \pm 3.05$ (vehicle) vs. $52.23 \pm 7.18$ (DAPT), $p<0.01)$.

\section{DAPT-treated induced membranes improve the bone} healing in rat femur but promote the bone resorption in subcutaneous site

To investigate whether DAPT-treated induced membranes were more favorable to bone formation. We applied both histological and imageological methods to evaluate the bone mass and the situation of bone resorption after 3 months autogenous bone graft. As is shown in Fig. 6, the density of bone volume in subcutaneous site was extremely lower than which in femoral defect site (Fig. 6b. Subcutaneous site, vehicle:28.16 \pm 6.08 DAPT: $19.82 \pm 4.05$; femoral defect site, vehicle: $47.57 \pm$ 5.63 DAPT: $66.82 \pm 8.93)$ and most residue bone are immature bone (Fig. 6c. Ratio of immature bone: vehicle: $68.21 \pm 10.51$ DAPT: $85.21 \pm 7.99)$. However, the bone grafts in femur were mature bone dominating (Fig. 6c. 

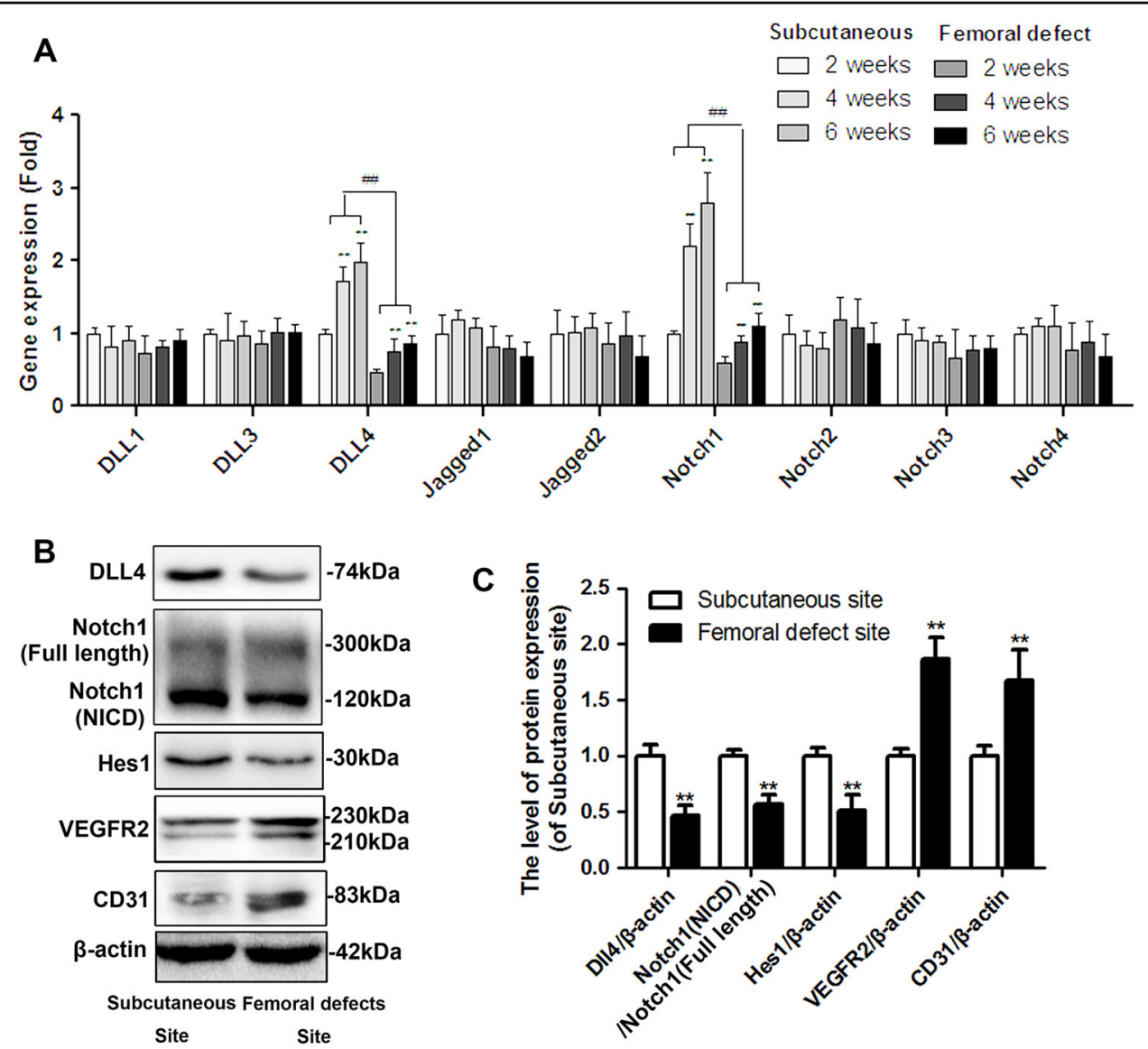

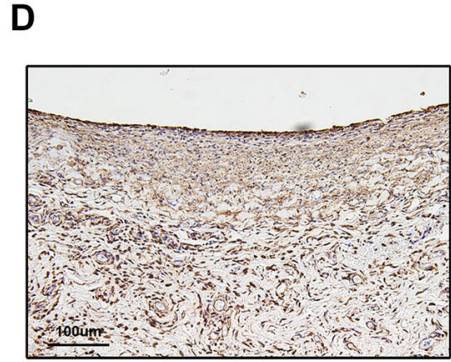

Subcutaneous site

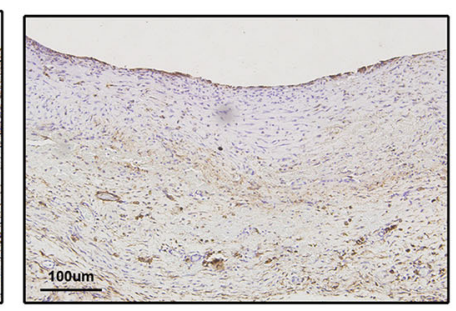

Femoral defects site

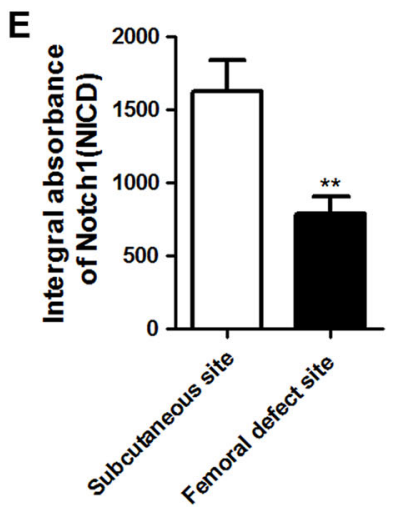

Fig. 3 The DIl4/Notch1 pathway in the induced membrane in the subcutaneous site and femoral defect site after 6 weeks of surgery. a The mRNA expression levels of 9 ligands or receptors involved in Notch signaling (ligands: DII1, DII3, DII4, Jagged1 and Jagged2; Receptors: Notch1, Notch2, Notch3 and Notch4) were screened by qRT-PCR in induced membrane, which formed in the subcutaneous site and femoral site at 2, 4, and 6 weeks ${ }^{* *} P<0.01$, vs. 2 -week induced membrane in the subcutaneous site and femoral defect site, respectively, ${ }^{\# \#} P<0.01$, vs. the same time point for the induced membrane in the subcutaneous site). b-c The protein expression of DII4, Notch1 (NICD), Hes1, VEGFR2, and CD31 in induced membrane derived from the subcutaneous site and femoral defect site. $\mathbf{d}$-e Immunohistochemistry staining of Notch1 (NICD) in induced membrane derived from the subcutaneous site and femoral defect site (scale bar: $50 \mu \mathrm{m}$ ). The data in the figures represent averages \pm S.D. Significant differences between the different groups are indicated as ${ }^{* *} P<0.01$, compared with induced membrane in the subcutaneous site; for each group, $n=5$

Ratio of immature bone: vehicle: $27.49 \pm 14.43$ DAPT: $25.27 \pm 8.52$ ). Meanwhile, DAPT-modified membrane showed less bone volume than vehicle group in subcutaneous environments (vehicle: $28.16 \pm 6.08$ vs. DAPT:
$19.82 \pm 4.05)$. Whereas, in femur site, DAPT group delineated a more compact and connective bone tissue with higher bone volume in comparing with vehicle group (vehicle: $47.57 \pm 5.63$ DAPT: $66.82 \pm 8.93$ ) and no 


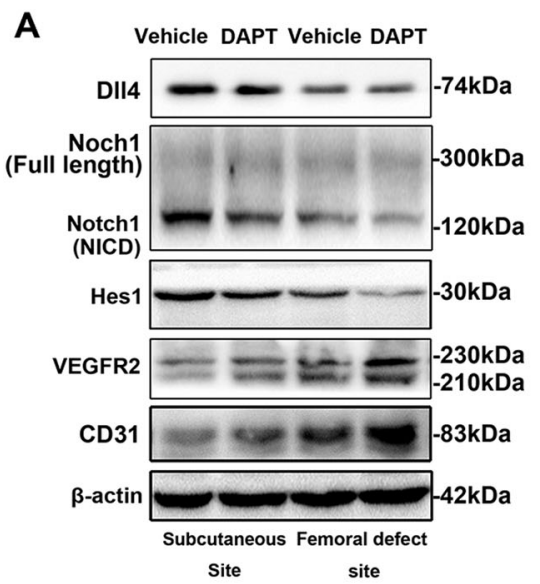

B
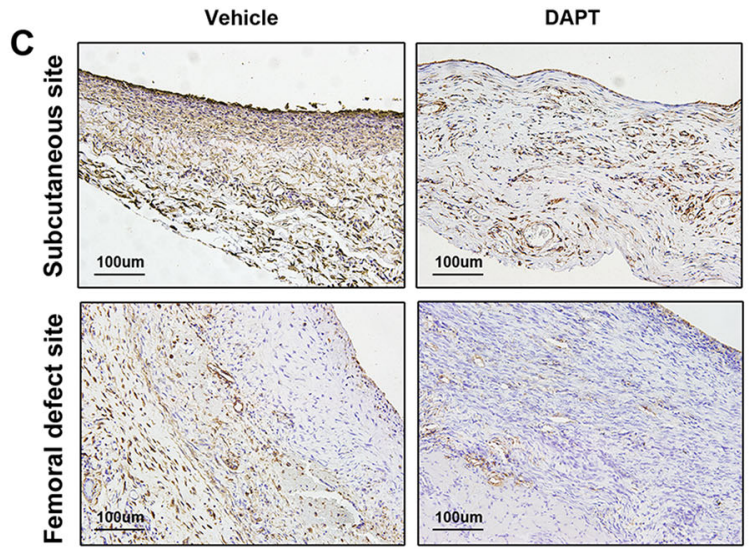

D
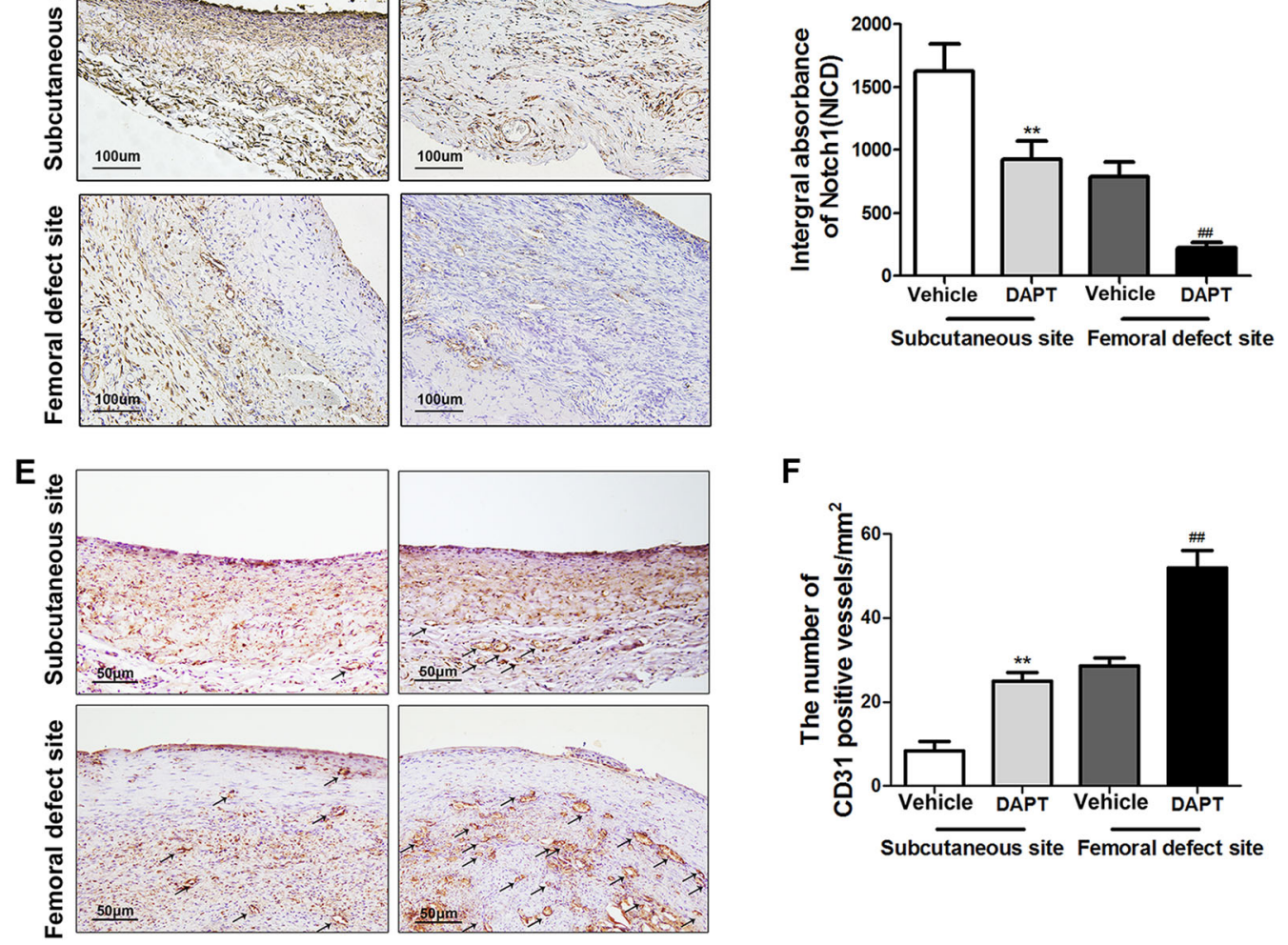

$\mathbf{F}$

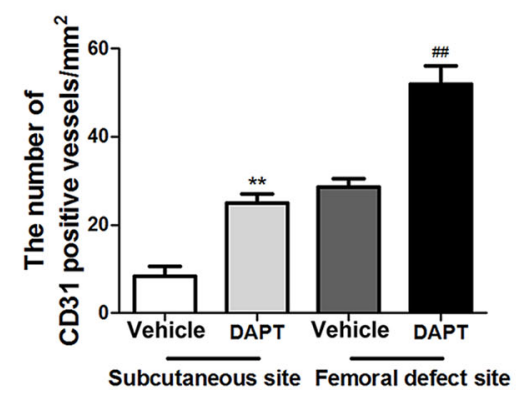

Fig. 4 Inhibition of Notch1 by DAPT promotes VEGFR2 and CD31 expression in the induced membrane in the subcutaneous site and femoral defect site. a-b Protein expression of DII4, Notch1 (NICD), Hes1, VEGFR2, and CD31 in induced membrane derived from the subcutaneous site and femoral defect site with or without DAPT intervention. c, e Immunohistochemistry staining of Notch1 (NICD) and CD31 in induced membrane derived from the subcutaneous site and femoral defect site with or without DAPT intervention (scale bar: $50 \mu m$ ). $\mathbf{d}, \mathbf{f}$ Quantitative results of the integral absorbance of the Notch1 and CD31-positive vessel density in induced membranes. The data in the figures represent averages \pm S.D. Significant differences between the different groups are indicated as ${ }^{* *} P<0.01$, compared with saline-treated induced membrane in the subcutaneous site; ${ }^{\# \#} P<0.01$, compared with saline-treated induced membrane in the femoral defect site; for each group, $n=5$

statistical significance of immature bone volume between these two groups (ratio of immature bone: vehicle: 27.49 \pm 14.43 DAPT: $25.27 \pm 8.52$ ). What is more, the bone regeneration situation in femur was also examined by $\mathrm{X}$ ray machine, the image revealed that the DAPT group not only possesed more bone volume than vehicle group, but also have a successive bridging callus formation between fracture end and grafts. However, the image of vehicle group exerted an obvious bone ununion in defect site (Fig. 6d). 

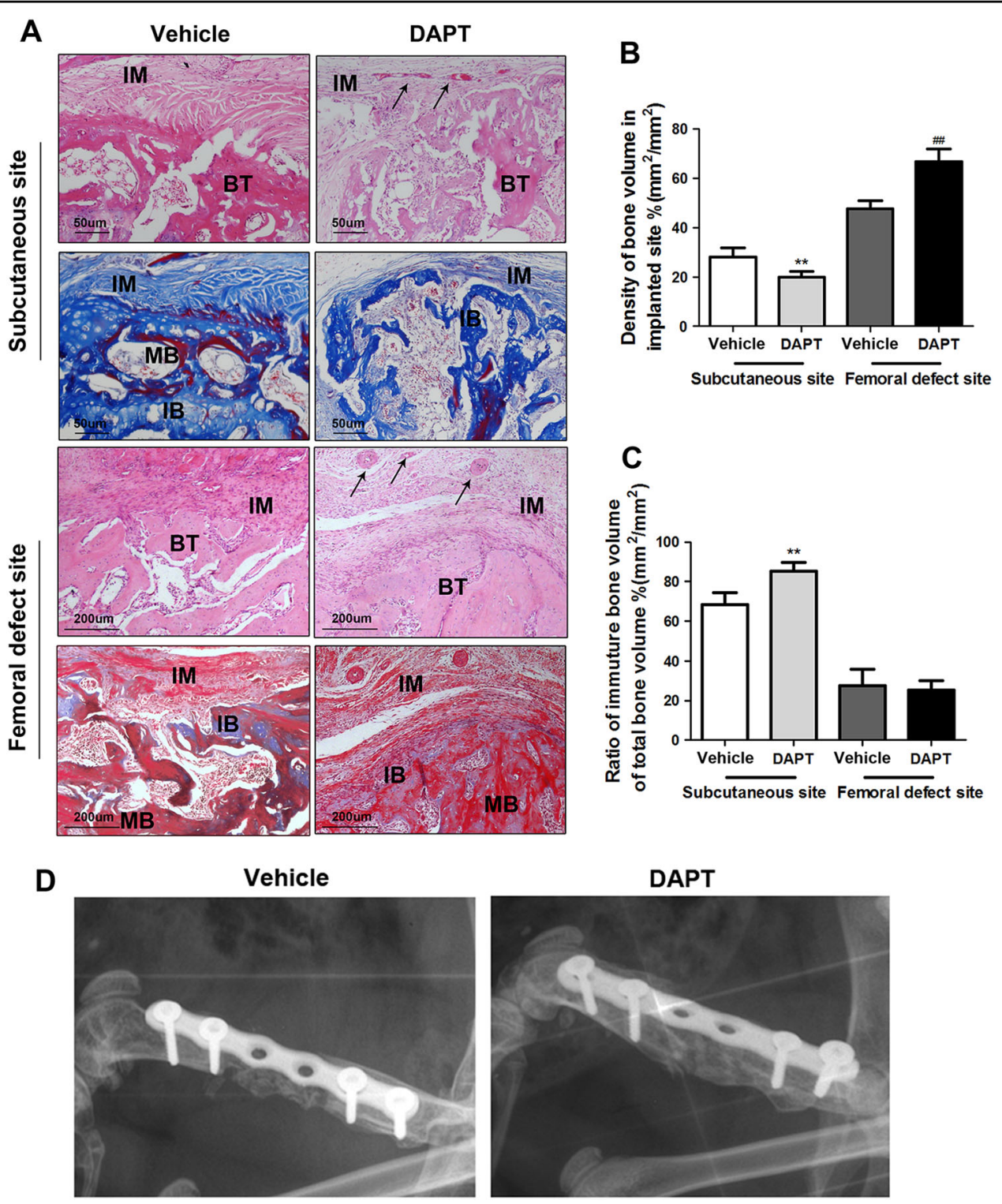

Fig. 5 DAPT-modified induced membrane promotes bone formation in the femoral defect site but induced graft resorption in subcutaneous site. a Representative image of H\&E staining and Masson's staining in the subcutaneous site and femoral defect site after 12 weeks of autogenous bone grafting (scale bar: $50 \mu \mathrm{m} / 200 \mu \mathrm{m}$, black arrows indicate microvessels, IM represents induced membrane, BT represents bone tissue, MB represents mature bone, IM represents immature bone). b Quantitative results of the bone mass density in the implanted site. c Quantitative results of the ratio of immature bone volume to the total bone volume. The data in the figures represent averages \pm S.D. The data in the figures represent averages \pm S.D. Significant differences between the different groups are indicated as ${ }^{*} P<0.01$, compared with saline-treated induced membrane in the subcutaneous site; ${ }^{\# \#} P<0.01$, compared with saline-treated induced membrane in the femoral defect site. $\mathbf{d} X$-ray image of explanted femurs 12 weeks after the second surgical step. Bone graft harvested from caudal vertebrae was used to fill the cavity delineated by different induced membranes. Grafts in vehicle-treated rats showed no bridging callus formation between the fracture end and graft (white arrow). Successful bone union was achieved in the grafted femurs in DAPT-modified induced membrane. For each group, $n=5$

Isolation, cultivation, and characterization of mouse EPCs EPCs were isolated from rat bone marrow. The phenotype of EPCs was first determined by FACS analysis to analyze expression of specific cell markers (Fig. 7a). The findings illustrated the expression of endothelial markers (CD31, VEGFR2, vascular endothelial-cadherin (VE-cadherin), and endothelial nitric oxide synthase (eNOS)) in the majority of the EPC population and confirmed the absence of the monocytic marker CD45. Then, fluorescent staining of double-positive cells with 1,10-dioctadecyl-3,3,3,30,30-tetramethylindocarbocyanine (Dil-I)labeled acetylated low-density lipoprotein (Dil-acLDL; Biomedical Technologies, USA) uptake and fluorescein isothiocyanate (FITC)-labeled Ulex europaeus agglutinin 

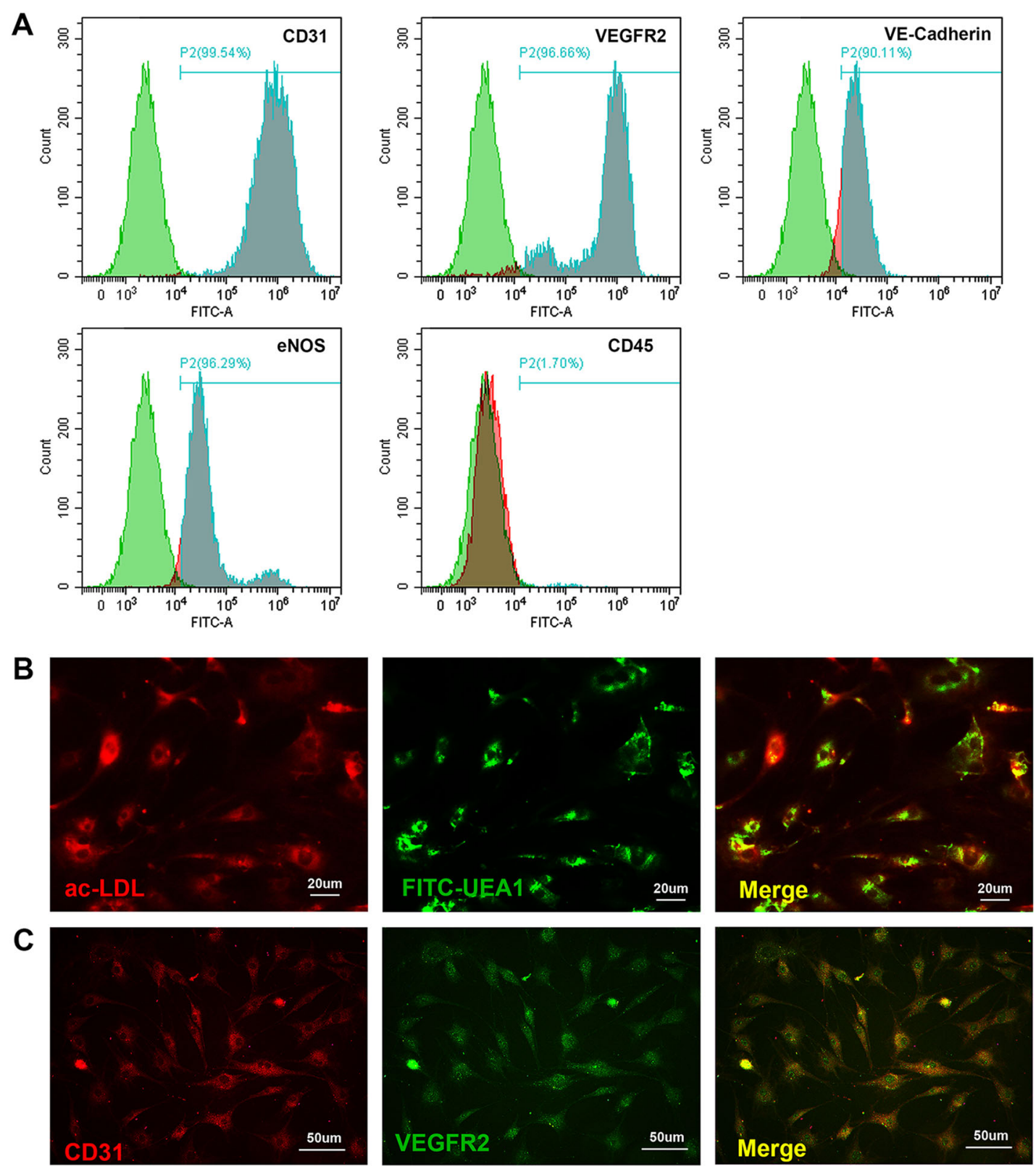

Fig. 6 Determination of the EPC phenotype. a FACS analysis was performed to assess the expression of endothelial markers in the EPC population, including CD31, VEGFR2, VE-cadherin and eNOS, as well as the monocytic marker CD45 b Fluorescent staining of double-positive cells with ac-LDL (red) uptake and FITC-UEA1 lectin binding (green) (scale bar: $20 \mu \mathrm{m}$ ). c Immunofluorescence staining of CD31 (red) and VEGFR2 (green) (scale bar: $50 \mu \mathrm{m})$

(UEA)-1 lectin (Vector Lab, USA) binding (Fig. 7b). Most cells were revealed to endocytose Dil-acLDL (red stained) as well as to bind FITC-labeled UEA-1 lectin (green stained). Furthermore, the immunofluorescence staining of specific EPCs markers (CD31 and VEGFR2) was performed to confirm the cells phenotype (Fig. 7c). The results illustrated the expression of endothelial markers in the majority of the EPCs population. Mature EPCs were collected and applied in the functional assays in present study.

\section{DAPT-treated induced membranes promote EPCs} proliferation, adhesion, recruitment, and angiogenic differentiation

To further explore whether DAPT-modified induced membranes have a positive effect on the angiogenesis of 

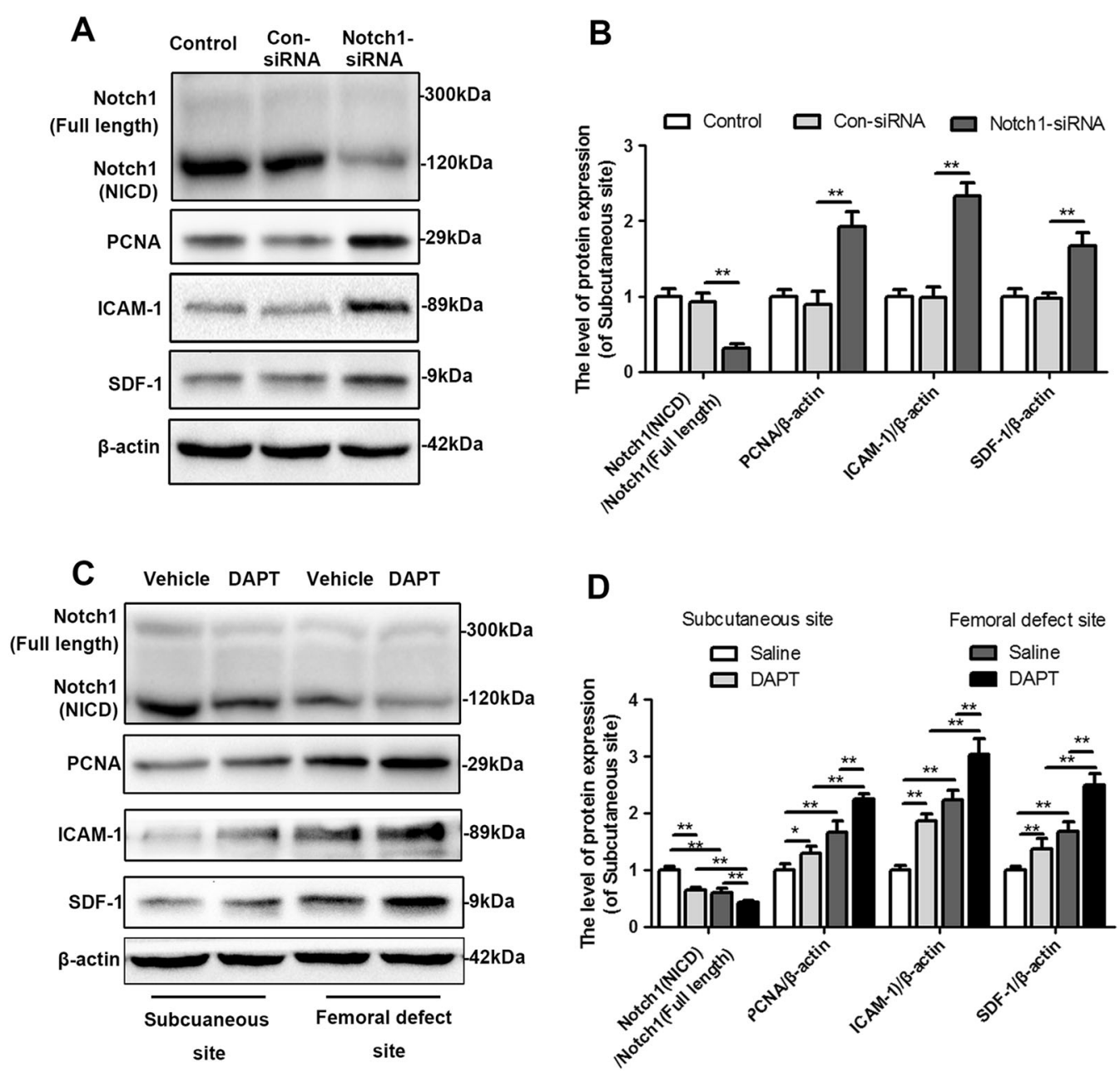

Fig. 7 Inhibition of Notch1 promotes proliferation, migration and recruitment of EPCs. a-b The protein expression and quantitative data for Notch1, PCNA, ICAM-1 and SDF-1 in EPCs treated with control-siRNA or Notch1-siRNA. c-d The protein expression and quantitative data for Notch1, PCNA, ICAM-1 and SDF-1 in EPCs treated with the protein extracted from the induced membrane derived from the subcutaneous site and femoral defect site with or without DAPT intervention. The data in the figures represent averages \pm S.D. Significant differences between the different groups are indicated as ${ }^{*} P<0.05,{ }^{*} P<0.01$; for each group, $n=5$

EPCs in vitro, the specific markers of endothelial cells proliferation (PCNA) $)^{36}$, adhesion (ICAM-1) ${ }^{37}$, and chemotaxis (SDF-1 $)^{38}$ were examined first by western blot analysis. As is shown in Fig. 8a, b, the EPCs treated by the protein extracted from femoral-induced membrane exert a higher expression of PCNA, ICAM-1, and SDF-1 than those treated by the protein extracted from subcutaneous. Meanwhile, proteins of DAPT-treated induced membrane also increased PCNA, ICAM-1, and SDF-1 expression of EPCs compared with the group treated by protein of membrane in vehicle group, which derived from both subcutaneous site and femoral defect site. What is more, the results of migration assay showed a dramatically increased ability to migrate in response to the protein of femoral-induced membrane or DAPT-treated membrane (Fig. 8c, f. Subcutaneous site, vehicle: $14.27 \pm 2.78$ DAPT:
$23.08 \pm 3.11$; femoral defect site, vehicle: $26.62 \pm 4.83$ DAPT: $38.12 \pm 5.27)$. And, according to the in vitro angiogenesis assay, protein collected from the membrane formed in femoral defect site or the membrane with DAPT intervention all increased the number of sprouting tubules compared with subcutaneous samples and saline treated group (Fig. 8d, f. Subcutaneous site, vehicle:3.16 \pm 1.06 DAPT: $6.06 \pm 1.21$; femoral defect site, vehicle: $8.95 \pm$ 2.01 DAPT: $13.87 \pm 2.72$ ). Similar results were founded in vitro invasion assay (Fig. 8e, h. Subcutaneous site, vehicle: $16.28 \pm 1.86$ DAPT: $19.32 \pm 2.85$; femoral defect site, vehicle: $27.39 \pm 4.55$ DAPT: $39.02 \pm 5.11$ ).

\section{Disscusion}

Masquelet's induced membrane technique occasionally discovered in 1986 by Masquelet, acts as a biological 


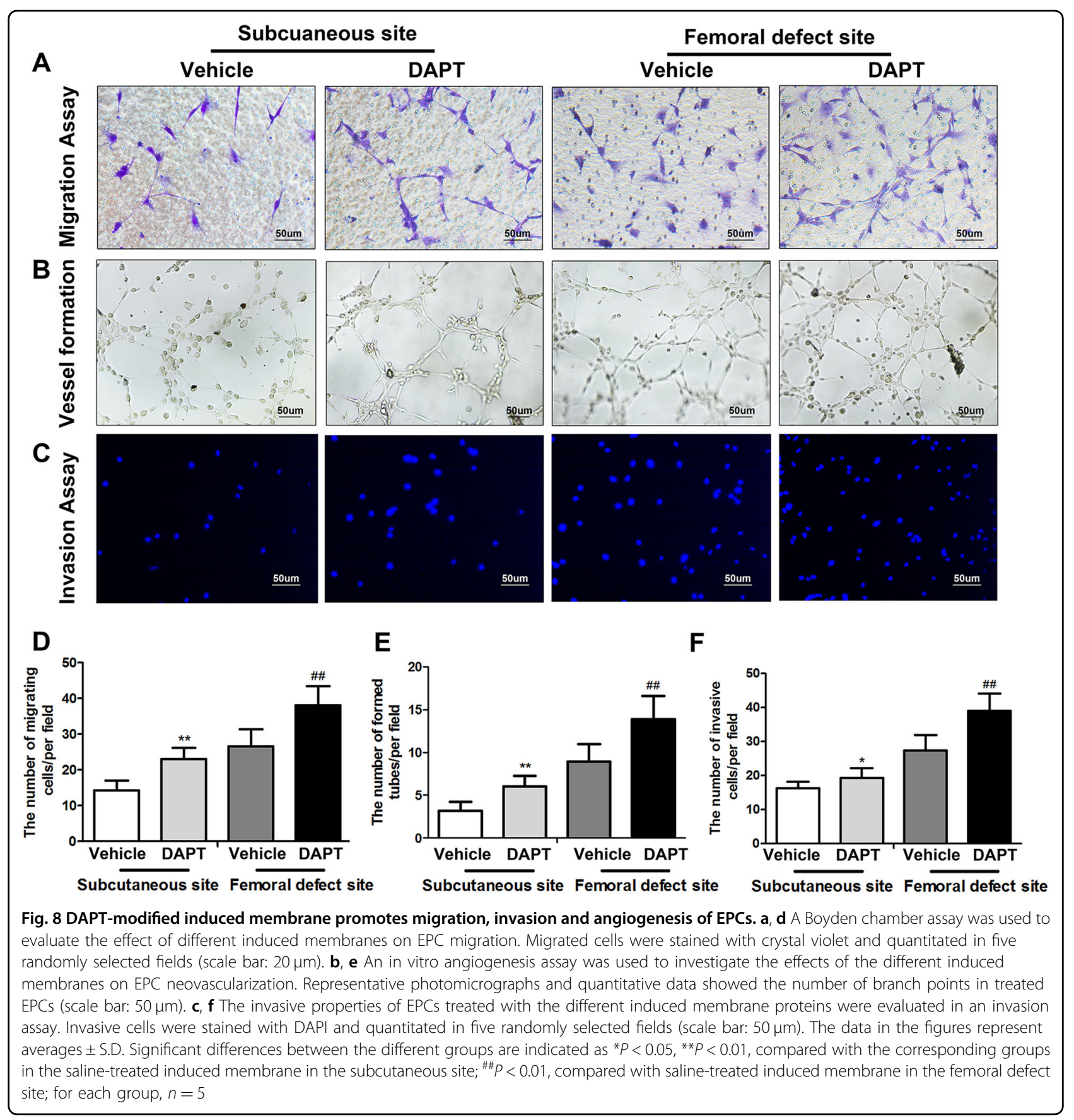

chamber to promote bone graft vascularity and corticalization while inhibit its resorption ${ }^{11}$. It opened a new perspective for bone reconstruction especially in the management of large scaled defect or septic segmental bone voids. Although such a functional membrane was applied in clinical practice for decades, there are still several weaknesses exist. Aho et al. reported that the vascularization of induced membrane got decreased after 6 weeks of membrane formation ${ }^{22}$. Meanwhile, the vascular axes of surrounded tissues contribute a lot to the membrane formation ${ }^{9}$. The cement spacer insert in femur or humerus defect showed a less vessels covered than in tibia defect, which would therefore delay the membrane mature ${ }^{9}$. It indicated that the segment with more vascular axes supplied would promote early vascularization and usability of the induced membrane. In addition, in case of an early bone graft implantation in the induced membrane, an immature and poorly vascularized tissue results in a lysis of the implanted bone graft. All of these studies suggested that promoting the neovascularization and 
angiogenesis of induced membrane is a promising strategy for membrane mature and anti-absorption of bone grafts, which consequently improved the successful rates of operation and reduced the duration of patients' hospitalization.

To our best knowledge of current studies, there are no former researches target the improvement of Masquelet's induced membrane itself, especially in the promotion of membranes vascularization. Herein, we first explored the role Dll4/Notch1 signaling pathway played in the induced membrane, which is a well-studied angiogenesis-related pathway. In the present in vivo studies, we found that the Dll4 and Notch1 were toughly in higher expression in 4 weeks and 6 weeks of induced membrane and especially in subcutaneous site, which was consistent with former H\&E results (Fig. 2) that the vessels density was lower in subcutaneous than in bone defect sites and decreased over time. It indicated that hindering Dll4/Notch1 might promote the vascularization of induced membrane. Then, DAPT, a classical $\gamma$-secretase inhibitor, was applied systemically to render the activity of Notch 1 expression. Although, the Dll4 expression was not altered after treatment, the activated Notch1 compound (NICD) as well as the downstream protein-like Hes1 were all dramatically inhibited and the membrane formed in both subcutaneous and bone defect exerts higher VEGFR2 and CD31 expression levels in DAPT treatment group than vehicle treatment group. Similar angiogenic effects of Notch inhibition were also reported in the studies of flap survival and wound healing ${ }^{33,34}$. However, these findings are in sharp contrast to the previous tumor angiogenesis studies in which Notch inhibition led to excessive and dysfunctional vasculature ${ }^{31}$. The reason for the difference results among these studies might due to the histological specificity of target tissues. In addition, it was interesting that the induced membrane with more vessels formation after DAPT intervention showed a more obvious resorption of bone grafts than vehicle-treated group. According to the Fig. 6a, the bone grafts in subcutaneous site are almost immature bone (blue staining), which indicated a potential demineralization process exist. It was well known that the process of osteogenesis in subcutaneous was also called ectopic bone formation. And the subcutaneous site is an inflammatory environment with amounts of osteoclasts or macrophagess infiltration to promote the bone graft resorption but with few osteoblasts or mesenchymal stem cells (MSCs) exist to make up for the bone lost ${ }^{39-41}$. With more blood supplement, the osteoclasts and macrophages were crucially activated to reduce the bone grafts. In contrast, abundant blood supplement would also recruit the bone marrow MSCs and promote its osteogenic differentiation to accelerate the bone formation in defect area.
In several former studies, the induced membrane was reported as a growth factor carrier, which could release angiogenic factors VEGF and vWF, as well as proteins associated with osteoblast proliferation and differentiation, like BMP-2 and TGF- $\beta 1^{18,42}$. Among these factors, VEGF is considered as a main factor that contributes to the vascularization ${ }^{43}$. The Dll4/Notch1 signaling is reported as a negative feedback loop to regulate exogenous VEGF secretion ${ }^{28,44,45}$. Besides, EPCs, a type of stem cells which would differentiate to mature endothelial cells, are frequently used in vitro studies of vessel formation ${ }^{46}$. Thus, we supplied EPCs with the protein extracted from DAPT-treated induced membrane to examine whether the membrane with Notch1 inhibition would improve the angiogenesis in vitro. As expected, the angiogenesis as well as the proliferation, migration, invasion, and adhesion of EPCs were all improved in DAPT-treated IM group compared with control one. These data suggested that the Notch1-inhibited environments were more favorable to the functional improvement of EPCs during the period of induced membrane formation.

In conclusion, our present study demonstrated that the induced membrane formed in subcutaneous showed a higher expression of Dll4/Notch1 signaling which was negatively associated with vessel density of induced membrane. Furthermore, promoting angiogenesis of induced membrane via Notch1 inhibition would improve the bone formation in bone defect site but result in grafts resorption in subcutaneous site. What is more, the induced membranes collected from subcutaneous and bone defect site in DAPT-treated rats both showed stronger angiogenic effects to EPCs compared with the membranes collected from vehicle group.

\section{Acknowledgements \\ This study was supported by National Natural Science Foundation of China (no. 81501869, no. 81601983, and no. 41506091), Medical and health science and technology project of Zhejiang Province (2016YKA139), and Zhejiang provincial Public welfare project (2017C33035).}

\section{Authors' contributions}

Conceived and designed the experiments: Q.T., H.L., and H.X. Performed the experiments: T.Q., H.J., and M.T. Analyzed the data: Z.X. and P.S. Contributed reagents/materials/analysis tools: Z.X., P.S., and L.S. Wrote the paper: Q.T., Y.Z., and H.L.

\section{Conflict of interest}

The authors declare that they have no conflict of interest.

\section{Publisher's note}

Springer Nature remains neutral with regard to jurisdictional claims in published maps and institutional affiliations.

Received: 16 September 2017 Revised: 30 December 2017 Accepted: 8 January 2018.

Published online: 20 April 2018 


\section{References}

1. Lasanianos, N. G., Kanakaris, N. K. \& Giannoudis, P. V. Current management of long bone large segmental defects. Orthop. Trauma 24, 149-163 (2010).

2. Apard, T. et al. Two-stage reconstruction of post-traumatic segmental tibia bone loss with nailing. Orthop. Traumatol. Surg. Res. 96, 549-553 (2010).

3. Bus, M. P. et al. Hemicortical resection and inlay allograft reconstruction for primary bone tumors: a retrospective evaluation in the Netherlands and review of the literature. J. Bone Jt. Surg. Am. 97, 738-750 (2015).

4. Mauffrey, C., Hake, M. E., Chadayammuri, V. \& Masquelet, A. C. Reconstruction of long bone infections using the induced membrane technique: tips and tricks. J. Orthop. Trauma 30, e188-e193 (2016).

5. Osterman, A. L. \& Bora, F. W. Free vascularized bone grafting for large-gap nonunion of long bones. Orthop. Clin. North Am. 15, 131-142 (1984).

6. Agarwal, R. \& Garcia, A. J. Biomaterial strategies for engineering implants for enhanced osseointegration and bone repair. Adv. Drug Deliv. Rev. 94, 53-62 (2015).

7. Aronson, J., Johnson, E. \& Harp, J. H. Local bone transportation for treatment of intercalary defects by the llizarov technique. Biomechanical and clinical considerations. Clin. Orthop. Relat. Res. 243, 71-79 (1989).

8. Han, W. et al. Induced membrane technique: advances in the management of bone defects. Int. J. Surg. 42, 110-116 (2017).

9. Auregan, J. C. \& Begue, T. Induced membrane for treatment of critical sized bone defect: a review of experimental and clinical experiences. Int. Orthop. 38, 1971-1978 (2014)

10. Masquelet, A. C., Fitoussi, F., Begue, T. \& Muller, G. P. Reconstruction of the long bones by the induced membrane and spongy autograft. Ann. Chir. Plast. Esthet. 45, 346-353 (2000).

11. Masquelet, A. C. \& Begue, T. The concept of induced membrane for reconstruction of long bone defects. Orthop. Clin. North Am. 41, 27-37 (2010)

12. Masquelet, A. C. \& Obert, L. Induced membrane technique for bone defects in the hand and wrist. Chir. Main 29, S221-S224 (2010).

13. Gan, A. W., Puhaindran, M. E. \& Pho, R. W. The reconstruction of large bone defects in the upper limb. Injury 44, 313-317 (2013).

14. Zwetyenga, N., Fricain, J. C., De Mones, E. \& Gindraux, F. Induced membrane technique in oral \& maxillofacial reconstruction. Rev. Stomatol. Chir. Maxillofac. 113, 231-238 (2012).

15. Bosemark, P., Perdikouri, C., Pelkonen, M., Isaksson, H. \& Tägil, M. The masquelet induced membrane technique with BMP and a synthetic scaffold can heal a rat femoral critical size defectc. J. Orthop. Res. 33, 488-495 (2015).

16. de Mones, E. et al. Comparative study of membranes induced by PMMA or silicone in rats, and influence of external radiotherapy. Acta Biomater. 19, 119-127 (2015).

17. Sasaki, G. et al. Induced membrane technique using beta-tricalcium phosphate for reconstruction of femoral and tibial segmental bone loss due to infection: technical tips and preliminary clinical results. Int. Orthop. 42, 17-24 (2018).

18. Henrich, D. et al. Establishment and characterization of the Masquelet induced membrane technique in a rat femur critical-sized defect model. J. Tissue Eng. Regen. Med. 10, E382-E396 (2016).

19. Villemagne, T. et al. Intercalary segmental reconstruction of long bones after malignant bone tumor resection using primary methyl methacrylate cement spacer interposition and secondary bone grafting: the induced membrane technique. J. Pediatr. Orthop. 31, 570-576 (2011).

20. Vigouroux, F. et al. Vascularised fibula or induced membrane to treat congenital pseudarthrosis of the Tibia: A multicentre study of 18 patients with a mean 9.5-year follow-up. Orthop. Traumatol. Surg. Res. 103, 747-753 (2017).

21. Liu, $H$. et al. Histological characteristics of induced membranes in subcutaneous, intramuscular sites and bone defect. Orthop. Traumatol. Surg. Res. 99, 959-964 (2013).

22. Aho, O. M. et al. The mechanism of action of induced membranes in bone repair. J. Bone Jt. Surg. Am. 95, 597-604 (2013).
23. Liu, Z. J. et al. Regulation of Notch1 and D\|l4 by vascular endothelial growth factor in arterial endothelial cells: implications for modulating arteriogenesis and angiogenesis. Mol. Cell Biol. 23, 14-25 (2003).

24. Sainson, R. C. et al. Cell-autonomous notch signaling regulates endothelial cell branching and proliferation during vascular tubulogenesis. FASEB J. 19, 1027-1029 (2005)

25. Takeshita, K. et al. Critical role of endothelial Notch1 signaling in postnatal angiogenesis. Circ. Res. 100, 70-78 (2007).

26. Hellstrom, M. et al. Dll4 signalling through Notch1 regulates formation of tip cells during angiogenesis. Nature 445, 776-780 (2007).

27. Coultas, L., Chawengsaksophak, K. \& Rossant, J. Endothelial cells and VEGF in vascular development. Nature 438, 937-945 (2005).

28. Bray, S. J. Notch signalling: a simple pathway becomes complex. Nat. Rev. Mol. Cell Biol. 7, 678-689 (2006)

29. Hofmann, J. J. \& Iruela-Arispe, M. L. Notch signaling in blood vessels: who is talking to whom about what? Circ. Res. 100, 1556-1568 (2007).

30. Ridgway, J. et al. Inhibition of Dll4 signalling inhibits tumour growth by deregulating angiogenesis. Nature 444, 1083-1087 (2006).

31. Noguera-Troise, I. et al. Blockade of Dll4 inhibits tumour growth by promoting non-productive angiogenesis. Nature 444, 1032-1037 (2006).

32. Williams, C. K., Li, J. L., Murga, M., Harris, A. L. \& Tosato, G. Up-regulation of the Notch ligand Delta-like 4 inhibits VEGF-induced endothelial cell function. Blood 107, 931-939 (2006).

33. Abbas, O. L. et al. Inhibition of the Notch pathway promotes flap survival by inducing functional neoangiogenesis. Ann. Plast. Surg. 75, 455-462 (2015).

34. Chigurupati, S. et al. Involvement of notch signaling in wound healing. PLoS ONE 2, e1167 (2007).

35. Li, S. et al. DAPT protects brain against cerebral ischemia by down-regulating the expression of Notch 1 and Nuclear factor kappa B in rats. Neurol. Sci. 33 1257-1264 (2012)

36. Tobler, K. et al. Reduction of both number and proliferative activity of human endothelial progenitor cells in obesity. Int. J. Obes. 34, 687-700 (2010).

37. $\mathrm{Wu}, \mathrm{Y}$. et al. Essential role of ICAM-1/CD18 in mediating EPC recruitment, angiogenesis, and repair to the infarcted myocardium. Circ. Res. 99, 315-322 (2006).

38. Odent Grigorescu, G. et al. Synergic effects of VEGF-A and SDF-1 on the angiogenic properties of endothelial progenitor cells. J. Tissue Eng. Regen. Med. 11, 3241-3252 (2017).

39. Glowacki, J., Rey, C., Cox, K. \& Lian, J. Effects of bone matrix components on osteoclast differentiation. Connect. Tissue Res. 20, 121-129 (1989).

40. Quinn, J., Joyner, C., Triffitt, J. T. \& Athanasou, N. A. Polymethylmethacrylateinduced inflammatory macrophages resorb bone. J. Bone Jt. Surg. Br. 74, 652-658 (1992).

41. Bruder, S. P., Fink, D. J. \& Caplan, A. I. Mesenchymal stem cells in bone development, bone repair, and skeletal regeneration therapy. J. Cell Biochem. 56, 283-294 (1994)

42. Pelissier, P., Masquelet, A. C., Bareille, R., Pelissier, S. M. \& Amedee, J. Induced membranes secrete growth factors including vascular and osteoinductive factors and could stimulate bone regeneration. J. Orthop. Res. 22, 73-79 (2004).

43. Ferrara, N., Gerber, H. P. \& LeCouter, J. The biology of VEGF and its receptors, Nat. Med. 9, 669-676 (2003).

44. Lobov, I. B. et al. Delta-like ligand 4 (D\|4) is induced by VEGF as a negative regulator of angiogenic sprouting. Proc. Natl Acad. Sci. USA 104, 3219-3224 (2007).

45. Gridley, T. Notch signaling in vascular development and physiology. Development 134, 2709-2718 (2007)

46. Asahara, T. et al. Bone marrow origin of endothelial progenitor cells responsible for postnatal vasculogenesis in physiological and pathological neovascularization. Circ. Res. 85, 221-228 (1999). 